\title{
Determination of the masses of the components of the HDE 226868/Cyg X-1 binary system
}

\author{
Janusz Ziolkowski ${ }^{* \dagger}$ \\ Copernicus Astronomical Center, Warsaw, Poland \\ E-mail: izecamk.edu.pl]
}

\begin{abstract}
Recent determination of the distance to HDE 226868/Cyg X-1 binary system (Reid et al., 2011) and more precise determination of the effective temperature of HDE 226868 (Caballero-Nieves et al., 2009) permit a more accurate estimate of the masses of both components. Using up to date evolutionary models, I obtain about $30 \pm 5 \mathrm{M}_{\odot}$ for the mass of the supergiant and about $18 \pm 5$ $\mathrm{M}_{\odot}$ for the mass of the black hole. Accepting rather liberal estimates of uncertainties in both the distance and the effective temperature, one gets ranges of 21 to $35 \mathrm{M}_{\odot}$ and 10 to $23 \mathrm{M}_{\odot}$ for both masses, respectively. The obtained mass of black hole agrees with the value $15 \pm 1 \mathrm{M}_{\odot}$ suggested by Orosz et al. (2011). However, the value suggested by them for the mass of the supergiant (19 $\pm 2 \mathrm{M}_{\odot}$ ) is wrong since it violates the mass-luminosity relation for the the massive MS stars (this relation was ignored in the iterative process of Orosz et al.).
\end{abstract}

An INTEGRAL view of the high-energy sky (the first 10 years) - 9th INTEGRAL Workshop and celebration of the 10th anniversary of the launch

15-19 October 2012

Bibliotheque Nationale de France, Paris, France

* Speaker.

${ }^{\dagger}$ A footnote may follow. 


\section{Introduction}

Cyg X-1 was the first recognized black hole (Bolton 1972) but its mass is still a subject of controversy. The long history of its determinations prior to 2005 is given by Ziolkowski (2005). After 2005, two major observational improvements, crucial for mass determination, took place. First, Caballero-Nieves et al. (2009, hereafter C-N+09) through careful stellar atmosphere modeling obtained more precise estimate of the effective temperature of supergiant HDE 226868, which is a binary companion of Cyg X-1. Second, Reid et al. (2011) estimated (through a radio parallax) the distance to the binary system HDE 226868/Cyg X-1. Orosz et al. (2011, hereafter O+11) used this distance to estimate the parameters of the binary system. They have done a very precise job, using 8 free parameters and fitting almost 600 observables: 3 UBV light curves (20 points each) and radial velocity curve (529 points). The values of the parameters were determined through an iterative scheme. The additional free parameter was the effective temperature of the supergiant. This parameter, however, was not iterated with the others, but was adjusted separately (the preferred value was found to be $T_{\mathrm{e}}=31000 \mathrm{~K}$ ). Allowing for non-synchronous rotation of the supergiant and slightly eccentric orbit, Orosz et al. were able to obtain impressively good fits of all three light curves and of the radial velocity curve. From their iterative process, they got $i=27.06 \pm 0.76^{\circ}$ for the binary orbit inclination and $M_{\mathrm{opt}}=19.16 \pm 1.90 \mathrm{M}_{\odot}$ and $M_{\mathrm{X}}=14.81 \pm 0.98 \mathrm{M}_{\odot}$ for the masses of both components. The formal errors are impressively low. However, the solution has a flaw: the mass of the optical component is inconsistent with its calculated luminosity $\left(\log L / \mathrm{L}_{\odot}=\right.$ 5.352). The mass and the luminosity of massive core hydrogen burning stars are not independent parameters, as I will demonstrate in this paper. I will also discuss the consequences of this fact.

\section{The Evolutionary Calculations for HDE 226868}

I calculated new evolutionary models for the supergiant HDE 226868, making use of a new more precise estimate of its effective temperature $(\mathrm{C}-\mathrm{N}+09)$ and of the just determined distance to the binary system (Reid et al 2011). The evolutionary tracks were computed for core hydrogenburning phase of stars with initial masses in the range $25-40 \mathrm{M}_{\odot}$. The Warsaw evolutionary code described by Ziolkowski (2005) was used. An initial chemical composition of $X=0.7$ and $Z=0.02$ was adopted.

The calculations were carried out under the assumption that the evolution starts from homogeneous configurations. Ziolkowski (2005) gave the arguments indicating that this is a good approximation. To calculate the stellar wind mass loss, I applied the commonly used formula derived by Hurley, Pols \& Tout (2000, hereafter HPT), based on parametrization of Nieuwenhuijzen \& de Jager (1990). As in earlier papers, I introduced the multiplicative factor $f_{\mathrm{SW}}$ applied to the HPT formula. The evolutionary calculations indicate, that to account for the evolutionary state of HDE 226868 , with the present day observational data, one has to use the value of $f_{\mathrm{SW}}$ in the range 2 to 5 .

\subsection{The mass-luminosity relation for massive core hydrogen burning stars}

This relation for the value of the effective temperature $T_{\mathrm{e}}=31000 \mathrm{~K}$ (i.e. the value adopted by $\mathrm{O}+11$ ) is shown in Fig. 1. As one can see, this relation is quite tight and the dependence on the 


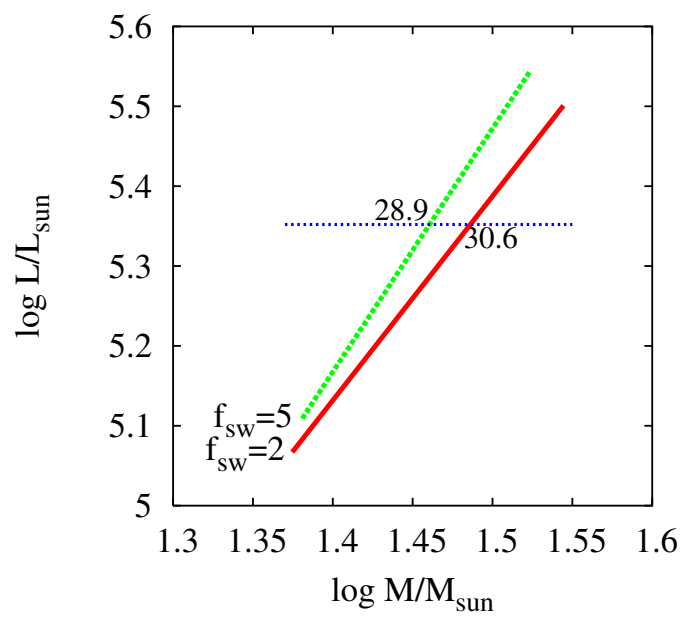

Figure 1: The mass-luminosity relation for massive core hydrogen burning stars at effective temperature $T_{\mathrm{e}}$ $=31000 \mathrm{~K}$. The relation is given for two values of the multiplying factor $f_{\mathrm{SW}}$ applied to HPT formula. The dotted horizontal line corresponds to the luminosity favored by O+11 solution. The values of the masses corresponding to the crossings of this line with $\mathrm{M}-\mathrm{L}$ relations are also shown (in solar units). Note that the value of the optical component mass suggested by $\mathrm{O}+11\left(19.2 \mathrm{M}_{\odot}\right)$ lies out of the frame of the picture.

uncertain parameter $f_{\mathrm{SW}}$ is weak. Please, note that the value of the supergiant mass suggested by O+11 $\left(19.2 \mathrm{M}_{\odot}\right)$ for their value of its luminosity $\left(\log L / \mathrm{L}_{\odot}=5.352\right)$ lies out of the frame of the picture.

\subsection{The evolutionary tracks in the Hertzsprung-Russell (HR) diagram}

The observational constraints given by C-N+09 for the effective temperature of HDE 226868 and by Reid et al. (2011) for the distance to the binary system produce an error box in the HR diagram, that is shown as a parallelogram in Fig. 2. The "best" observational solution lies in the center of this parallelogram. The solution advocated by $\mathrm{O}+11$ is shown as a yellow cross. The procedure of obtaining the different evolutionary models of HDE 226868 was the following one. For each pair of the chosen values of $T_{\mathrm{e}}$ and $d$ (corresponding e.g. to the center or to a corner of the error box) a corresponding position (i.e. a pair of the values of $T_{\mathrm{e}}$ and $L$ ) was calculated. Then an evolutionary track passing through that position was calculated. The value of $f_{\mathrm{SW}}$ was adjusted so as to obtain a stellar wind strength at tis position consistent within $10 \%$ with the observed value $\left(\dot{M}=-2.6 \times 10^{-6} \mathrm{M}_{\odot} / \mathrm{yr}\right.$, Gies \& al. 2003).

This procedure produced several possible evolutionary models of HDE 2268668 that are listed in Tab. 1. The corresponding evolutionary tracks in the HR diagram are shown in Fig. 2.

As may be seen, assuming wide margins for uncertainty of both $T_{\mathrm{e}}$ and $d$, one gets the mass of HDE 226868 in the range 20.7 to $35.4 \mathrm{M}_{\odot}$. However, I believe that the value of $T_{\mathrm{e}}$ equal $25500 \mathrm{~K}$ is rather too low for HDE 226868. Also, the value $f_{\mathrm{SW}}=8$, required to get a fit for this temperature, seems to be excessively high. Therefore, I would rather discard the third line in Tab. 1 and state that, on evolutionary ground, the mass of HDE 226868 is probably in the range 25 to $35 \mathrm{M}_{\odot}$. 


\begin{tabular}{|c|r|r|r|l|l|}
\hline $\begin{array}{c}d \\
{[\mathrm{kpc}]}\end{array}$ & $\begin{array}{c}T_{\mathrm{e}} \\
{\left[10^{3} \mathrm{~K}\right]}\end{array}$ & $\begin{array}{c}\log L \\
{\left[\mathrm{~L}_{\odot}\right]}\end{array}$ & $\begin{array}{c}M_{\mathrm{opt}} \\
{\left[\mathrm{M}_{\odot}\right]}\end{array}$ & $f_{\mathrm{SW}}$ & $\begin{array}{c}M_{0} \\
{\left[\mathrm{M}_{\odot}\right]}\end{array}$ \\
\hline & & & & & \\
1.86 & 28.0 & 5.309 & 27.2 & 3.6 & 33 \\
1.98 & 30.5 & 5.513 & 35.4 & 1.76 & 40 \\
1.75 & 25.5 & 5.094 & 20.7 & 8 & 29 \\
1.75 & 28.0 & 5.257 & 25.5 & 4.9 & 32.5 \\
1.86 & 31.0 & 5.352 & 29.4 & 4 & 36 \\
& & & & & \\
\hline
\end{tabular}

Table 1: Mass of the optical component for the different assumed values of its effective temperature and of the distance to the binary system.

NOTE:

$f_{\mathrm{SW}}$ denotes the multiplying factor applied to HPT formula; $M_{0}$ denotes the initial (ZAMS) mass of the optical component for a given evolutionary track; other symbols have their usual meanings

\section{The mass of the black hole component}

Once the evolutionary model of the supergiant component is selected from Tab. 1, we can use two equations discussed by Ziolkowski (2005):

$$
\begin{gathered}
M_{\mathrm{opt}} \sin ^{3} i /\left[q(1+q)^{2}\right]=0.251, \\
R_{\mathrm{opt}}=f_{\mathrm{RL}}(0.38+0.2 \log q)(1+q) \times 8.36 / \sin i .
\end{gathered}
$$

where $R_{\text {opt }}$ is the radius of $\operatorname{HDE} 226868, f_{\mathrm{RL}}$ is the fill-out factor $\left(f_{\mathrm{RL}}=R_{\mathrm{opt}} / R_{\mathrm{RL}}\right.$, where $R_{\mathrm{RL}}$ is the radius of the Roche lobe around HDE 226868), $q=M_{\mathrm{opt}} / M_{\mathrm{X}}$ is the ratio of the masses of the components and $i$ is the inclination of the orbit.

Once we assume the value of the coefficient $f_{\mathrm{RL}}$, we can solve the the eqs. (3.1)-(3.2) for $i$ and $q$ and then get also the mass of the compact component $M_{\mathrm{X}}$.

We do not have large freedom in selecting the value of $f_{\mathrm{RL}}$. Earlier observations and analysis (Gies \& Bolton 1986a,b; C-N+09; O+11) indicate that it has to be larger than 0.9 and, most likely, not smaller than 0.95 . Similarly, not all resulting values of the inclination $i$ are acceptable. The same observations and analysis indicate that it cannot be substantially different from about $30^{\circ}$ $\left(\mathrm{O}+11\right.$ got $i \sim 27^{\circ}$.

Models of the binary system, obtained by taking evolutionary supergiant models from Tab.1 and selecting the value of $f_{\mathrm{RL}}$ in the range $0.95-1$ are listed in Tab. 2. 


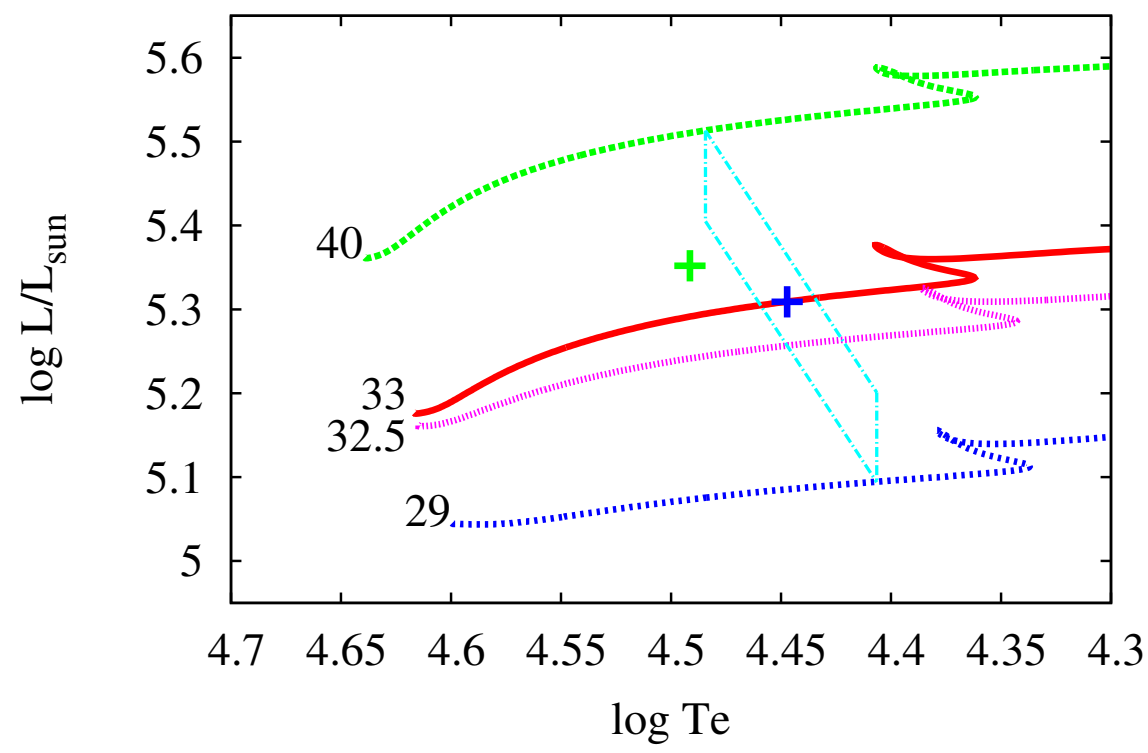

Figure 2: The evolutionary tracks in the H-R diagram. The tracks are labeled with the initial mass of the star (in solar units). The values of the multiplying factor $f_{\mathrm{SW}}$ applied to HPT formula were adjusted individually for each track (see Table 1), so as to obtain agreement with the observed value of the present strength of stellar wind from HDE 226868. The tracks were meant to reproduce the observed values of the present luminosity and effective temperature of HDE 226868 according to the different solutions listed in Table 1. The parallelogram describe the observational error box using effective temperature estimates of $\mathrm{C}-\mathrm{N}+09$ and distance estimates of $\mathrm{R}+11$ (the blue cross lies in its center). The green cross corresponds to $\mathrm{O}+11$ solution.

The last two lines of this table should be discarded, since they require too small value of $f_{\mathrm{RL}}$. Taken at face value, Tab. 2 imply the mass of the black hole in the range 10 to $23 \mathrm{M}_{\odot}$. However, if one remembers what was said about the realistic supergiant models (section 2.2) and if one requires additionally that $f_{\mathrm{RL}}$ is $\geq 0.95$, then one gets the range $13-23 \mathrm{M}_{\odot}$. I consider this the most likely range for the mass of the black hole in the system.

\section{Acknowledgments}

I would like to thank A. Zdziarski for a careful reading of the manuscript and for helpful comments. This work was partially supported by the Polish Ministry of Science and Higher Education (MSHE) project 362/1/N-INTEGRAL (2009-2012) and by MSHE grant N N203 581240 (2011-2014) to A. Zdziarski.

\section{References}

[1] C.T. Bolton, Identification of Cygnus X-1 with HDE 226868, Nature 235 (1972) 271

[2] S.M. Caballero-Nieves, D.R. Gies, C.T. Bolton, P. Hadrava, A. Herrero, T.C. Hillwig, S.B. Howell, W. Huang, L. Kaper, P. Koubsky \& M.V. McSwain, The Ultraviolet Spectrum and Physical Properties of the Mass Donor Star in HD 226868 = Cygnus X-1, ApJ 701 (2009) 1895 (C-N+09) 


\begin{tabular}{|c|r|r|c|c|c|c|}
\hline $\begin{array}{c}d \\
{[\mathrm{kpc}]}\end{array}$ & $\begin{array}{c}T_{\mathrm{e}} \\
{\left[10^{3} \mathrm{~K}\right]}\end{array}$ & $\begin{array}{c}\log L \\
{\left[\mathrm{~L}_{\odot}\right]}\end{array}$ & $\begin{array}{c}M_{\mathrm{opt}} \\
{\left[\mathrm{M}_{\odot}\right]}\end{array}$ & $f_{\mathrm{RL}}$ & $\begin{array}{c}i \\
{\left[{ }^{0}\right]}\end{array}$ & $\begin{array}{c}M_{\mathrm{x}} \\
{\left[\mathrm{M}_{\odot}\right]}\end{array}$ \\
\hline \multirow{3}{*}{1.86} & 28.0 & 5.309 & 27.2 & 0.95 & 34.4 & 13.1 \\
& & & & 0.98 & 25.0 & 19.3 \\
1.98 & 30.5 & 5.513 & 35.4 & 0.92 & 33.8 & 15.6 \\
& & & & 0.95 & 24.5 & 22.9 \\
1.75 & 25.5 & 5.094 & 20.7 & 0.98 & 38.3 & 10.0 \\
& & & & 1.00 & 31.0 & 12.7 \\
1.75 & 28.0 & 5.257 & 25.5 & 0.91 & 37.0 & 11.7 \\
& & & & 0.95 & 23.7 & 20.0 \\
1.86 & 31.0 & 5.352 & 29.4 & 0.80 & 31.9 & 14.9 \\
& & & & 0.83 & 21.8 & 24.1 \\
& & & & & & \\
\hline
\end{tabular}

Table 2: Parameters of the selected evolutionary models of the binary system HDE 226868/Cyg X-1.

NOTE:

$f_{\mathrm{RL}}$ denotes the Roche lobe fill-out factor; $i$ denotes the inclination of the binary orbit; other symbols have their usual meanings

[3] D.R. Gies \& C.T. Bolton, The optical spectrum of HDE $226868=$ Cygnus X-1. II. Spectrophotometry and mass estimates, ApJ 304 (1986a) 371

[4] D.R. Gies \& C.T. Bolton, The optical spectrum of HDE $226868=$ Cygnus X-1. III. A Focused Stellar Wind Model for He II lambda 4686 Emission, ApJ 304 (1986b) 389

[5] D.R. Gies, C.T. Bolton, Wind Accretion and State Transitions in Cygnus X-1, ApJ 583 (2003) 424

[6] J.R. Hurley, O.R. Pols \& C.A. Tout, Comprehensive analytic formulae for stellar evolution as a function of mass and metallicity, MNRAS 315 (2000) 543 (HPT)

[7] H. Nieuwenhuijzen \& C. de Jager, it Parametrization of stellar rates of mass loss as functions of the fundamental stellar parameters M, L, and R, A\&A 231 (1990) 134

[8] J.A. Orosz, J.E. McClintock, J.P. Aufdenberg, R.A. Remillard, M.J. Reid, R. Narayan \& L. Gou, The Mass of the Black Hole in Cygnus X-1, ApJ 742 (2011) 84 (O+11)

[9] M.J. Reid, J.E. McClintock, R. Narayan, L. Gou, R.A. Remillard \& J.A. Orosz, The Trigonometric Parallax of Cygnus X-1, ApJ 742 (2011) 83

[10] J. Ziolkowski, Evolutionary constraints on the masses of the components of the HDE 226868/Cyg X-1 binary system, MNRAS 358 (2005) 851 